\title{
Avaliação antropométrica de crianças em creches do município de Bezerros, PE
}

\author{
Anthropometric evaluation of children from day care centers in the municipality of Bezerros, \\ Pernambuco, Brazil
}

Cláudia Porto S. Pinho ${ }^{1}$, Jeymison Emanoel M. da Silva², Ana Carla G. Silva², Nayara Nagry A. de Araújo², Carolina Estevam

Fernandes ${ }^{3}$, Fernanda Cristina de L. Pinto ${ }^{4}$

\section{RESUMO}

Objetivo: Avaliar o estado nutricional de crianças matriculadas em creches da rede pública de ensino do município de Bezerros, Pernambuco.

Métodos: Estudo transversal que analisou os indicadores antropométricos de 735 crianças com idade entre um e sete anos (70\% das matrículas do ano letivo de 2008 no município). Para classificação do estado nutricional, foram utilizados os índices estatura/idade (E/I) e peso/estatura $(\mathrm{P} / \mathrm{E})$, expressos em valores de escore $\mathrm{Z}$ com base na curva do CDC (2000). O software utilizado para análise dos dados foi o Epi-Info, versão 6,04.

Resultados: Das crianças estudadas, 53,7\% eram do sexo masculino e $72,7 \%$ provenientes de creches situadas em área urbana. A avaliação nutricional apontou uma prevalência de déficit nutricional de 6,8 e 0,7\% segundo os índices E/I e P/E, respectivamente. A prevalência de obesidade segundo o $\mathrm{P} / \mathrm{E}$ foi de $6,1 \%$. Houve maior prevalência de baixa estatura entre as crianças do sexo feminino e risco para baixa estatura para o sexo masculino. Não foram observadas diferenças no estado nutricional das crianças de creches de área urbana e rural. Das crianças com déficit de E/I, 10\% apresentaram obesidade associada. Calculando-se a razão entre a prevalência de obesidade e déficit de P/I, verificou-se razão de 1,7:1, sendo 1,3:1 e 5:1 para as crianças do meio urbano e rural, respectivamente.

Conclusões: A baixa estatura e a obesidade foram os distúrbios nutricionais mais prevalentes na população de

Instituição: Prefeitura Municipal de Bezerros, PE, Brasil

${ }^{1}$ Mestranda em Nutrição pela Universidade Federal de Pernambuco (UFPE); Nutricionista da Secretaria Municipal de Educação e Cultura de Bezerros, Recife, PE, Brasil

${ }^{2}$ Acadêmico do Curso de Nutrição da Faculdade Vale do Ipojuca (Favip), Bezerros, PE, Brasil

${ }^{3}$ Doutoranda em Nutrição pela UFPE; Professora do Curso de Graduação em Nutrição da Favip, Recife, PE, Brasil

${ }^{4}$ Doutoranda em Nutrição pela UFPE; Professora Assistente da Universidade Federal da Paraíba (UFPB), João Pessoa, PB, Brasil estudo, com maior expressividade do processo de transição nutricional nas crianças de área rural.

Palavras-chave: criança; creches; antropometria; estado nutricional.

\section{ABSTRACT}

Objective: To evaluate the nutritional status of children from day care centers of the public educational system in the municipality of Bezerros, Pernambuco, Brazil.

Methods: This cross-sectional study examined the anthropometric indicators of 735 children aged between one and seven years old, which accounted for $70 \%$ of the children enrolled at the educational system of Bezerros municipality in 2008. The indicators used for classification of the nutritional status were height/age (H/A), weight/ age (W/A) and weight/height (W/H), all expressed as Zscore values. The software used for data analysis was the Epi-Info, version 6.04.

Results: In the studied group, $53.7 \%$ of the children were males and $72.7 \%$ were from day care centers located in the urban area. The nutritional assessment revealed a prevalence of nutritional deficit of 6.8 and $0.7 \%$ according to $\mathrm{H} / \mathrm{A}$ and $\mathrm{W} / \mathrm{H}$, respectively. The prevalence of obesity according to the $\mathrm{W} / \mathrm{H}$ was $6.1 \%$. A nutritional risk for short stature was observed among males, and a higher prevalence of short stature was verified among female children. There were no

\author{
Endereço para correspondência: \\ Cláudia Porto S. Pinho \\ Rua Bianor de Oliveira, 262 - Campo Grande \\ CEP 52040-350 - Recife/PE \\ E-mail: claudia_sabinopinho@yahoo.com.br \\ Fonte de financiamento: Prefeitura Municipal de Bezerros (PE) \\ Conflito de interesse: nada a declarar
}

Recebido em: 21/5/2009

Aprovado em: 7/12/2009 
differences between urban and rural day care centers in relation to children nutritional status. Obesity was associated to $\mathrm{H} / \mathrm{A}$ deficits in $10 \%$ of the children. The ratio between the prevalence of overweight and deficit of W/A was 1.7:1, being 1.3:1 for children from the urban areas and 5:1 for those from rural areas.

Conclusion: Short stature and obesity were the most prevalent nutritional disorders in the studied population. The nutritional transition process was more evident in children of rural area.

Key-words: child; child day care centers; anthropometry; nutritional status.

\section{Introdução}

A situação nutricional das crianças de um país é essencial para aferir a evolução das condições de saúde e de vida da população devido ao seu caráter multicausal e à sua relação com o grau de atendimento das necessidades básicas como alimentação, saneamento, acesso aos serviços de saúde, nível de renda e educação $0^{(1-4)}$.

Há evidências de que a condição nutricional da criança brasileira apresentou modificações substanciais nos últimos 15 anos, com redução da taxa de desnutrição e aumento da obesidade infantil ${ }^{(5)}$. A prevalência esperada de déficit nutricional ou excesso de peso em uma população com boas condições de saúde e nutrição é de $2,3 \%$, sendo que prevalências inferiores são interpretadas como ausência virtual de desvios nutricionais na população ${ }^{(1,6,7)}$.

$\mathrm{O}$ uso de índices antropométricos tem sido considerado uma estratégia válida para gerar indicadores sensíveis do estado nutricional ${ }^{(8)}$, particularmente durante a idade pré-escolar, por refletir as condições nutricionais e, indiretamente, as influências do ambiente socioeconômico ${ }^{(4)}$.

Alguns estudos têm chamado atenção para as vantagens da análise do estado nutricional de crianças a partir do espaço/ instituição que elas frequentam, como creches e escolas ${ }^{(9)}$. $\mathrm{O}$ interesse em conhecer a magnitude dos problemas nutricionais, tendo como unidade de diferenciação o espaço/ instituição que as crianças frequentam, reside na possibilidade de identificar a distribuição dos distúrbios nutricionais, monitorar desigualdades sociais em saúde e, especialmente, possibilitar a identificação de necessidades de implementação de ações específicas e diferenciadas de nutrição e saúde ${ }^{(8)}$. A Organização Mundial de Saúde (OMS) e o Ministério da Saúde (MS) reconhecem a importante influência das condições de vida sobre o crescimento, tornando o ambiente escolar ideal para a realização de atividades de atenção nutricional à criança $a^{(10)}$.

Bezerros é um município do agreste pernambucano com população estimada em 57.371 habitantes (22\% em área rural) ${ }^{(11)}$, índice de desenvolvimento humano (IDH) de 0,619, abaixo do IDH médio dos municípios brasileiros $(0,6993)$, e posição 4.312 no ranking nacional ${ }^{(12)}$, com incidência de pobreza de $53 \%{ }^{(11)}$. Nesse município, não há informações publicadas sobre o estado nutricional de crianças de escolas públicas.

Como os primeiros anos de vida são decisivos para o crescimento e desenvolvimento infantil e o acompanhamento do estado nutricional nessa fase fornece informações relevantes para avaliar a saúde e os riscos de morbimortalidade ${ }^{(13)}$, conhecer o estado nutricional das crianças que frequentam creches públicas é importante para subsidiar a formulação de estratégias para prevenção e controle dos distúrbios nutricionais que acometem a infância. Desta forma, o presente estudo teve o objetivo de avaliar o estado nutricional de crianças matriculadas nas creches da rede pública de ensino do município de Bezerros, em Pernambuco.

\section{Métodos}

Trata-se de um estudo transversal, utilizando levantamento de dados antropométricos de crianças de creches da rede pública de ensino do município de Bezerros (PE). A coleta de dados foi realizada no mês de abril de 2008, considerando-se todas as crianças na faixa etária de um a sete anos e matriculadas em alguma das oito creches do município, situadas em área urbana (quatro creches) ou rural (quatro creches).

Do total de 1.050 crianças matriculadas no ano letivo de 2008, 735 foram incluídas no estudo (70\%), sendo 395 do sexo masculino e 340 do sexo feminino. Foram excluídas aquelas que estavam ausentes no momento da coleta $(20,2 \%)$, que se desvincularam das creches até o período de realização da coleta de dados $(7,2 \%)$, e/ou que apresentaram dados incompletos quanto às variáveis estudadas (peso, idade, altura) $(2,6 \%)$, totalizando uma perda de $30 \%$. As perdas por ausência no momento da coleta poderiam ser minimizadas com retorno posterior às creches, entretanto, devido às dificuldades de recursos e de acesso geográfico (especialmente na área rural), esse procedimento não foi adotado.

No início da pesquisa, os dirigentes das creches foram esclarecidos quanto à metodologia adotada e as unidades 
escolares foram visitadas para verificar a rotina diária, a fim de operacionalizar as ações sem interferir no andamento normal das atividades. Os pais/responsáveis foram informados sobre os objetivos da pesquisa e assinaram termo de consentimento livre e esclarecido.

Os dados foram coletados por estagiários do curso de $\mathrm{Nu}-$ trição e as medidas antropométricas foram tomadas de acordo com as técnicas estabelecidas pela Organização Mundial da Saúde (OMS) ${ }^{(14)}$, com supervisão da nutricionista responsável pela pesquisa. As crianças apresentavam-se com o mínimo de roupas, sendo o peso verificado em balança antropométrica mecânica (marca Welmy ${ }^{\circledR}$ ) com capacidade para $150 \mathrm{~kg}$ e divisão de $100 \mathrm{~g}$. O comprimento das crianças com menos de 24 meses foi aferido com estadiômetro infantil, com precisão de $1 \mathrm{~cm}$. As crianças com idade superior a 24 meses foram medidas em pé, descalças, com a régua antropométrica acoplada à balança.

Os índices antropométricos utilizados neste estudo foram peso/idade $(\mathrm{P} / \mathrm{I})$, estatura/idade $(\mathrm{E} / \mathrm{I})$ e peso/estatura $(\mathrm{P} / \mathrm{E})$ medidos em valores de escores Z, com o software EPINUT, incluso no pacote Epi-Info 6.04, que apresenta como curva de referência a curva do Centers for Disease Control and Prevention (CDC, 2000).

Para classificar o estado nutricional, foram empregados os escores $\mathrm{Z}$ dos índices $\mathrm{E} / \mathrm{I}$ e $\mathrm{P} / \mathrm{E}$, adotando-se os critérios da OMS: escore $Z<-2$ referente à baixa estatura/ desnutrição; escore $Z$ entre -2 e -1 referente a risco de comprometimento estatural/nutricional; escore $Z$ entre -1 e +2 significando estatura adequada/eutrofia. Os valores de escore $\mathrm{Z} \geq+2$ relativos ao indicador $\mathrm{P} / \mathrm{E}$ foram definidos como obesidade.

$\mathrm{O}$ indicador utilizado para avaliar o processo de transição nutricional foi construído a partir da razão obesidade/ desnutrição, estabelecida pelo quociente entre a prevalência de obesidade (escore $Z$ de peso/estatura $\geq+2$ ) e a prevalência de déficit de peso (escore $Z$ de peso para idade $<-2$ ). Esse é um parâmetro que indica a transição nutricional a partir da observação do aumento da obesidade e decréscimo da desnutrição ${ }^{(2)}$.

Os dados foram analisados no software Epi-Info, versão 6.04, empregando-se o teste $t$ de Student para amostras nãopareadas para análise das médias com distribuição normal e qui-quadrado para análise das frequências, considerando significância estatística se $p<0,05$.

A pesquisa foi aprovada pelo Comitê de Ética em Pesquisa com Seres Humanos da Faculdade Vale do Ipojuca.

\section{Resultados}

Dentre as 735 crianças avaliadas, $53,7 \%$ eram do sexo masculino e $72,7 \%$ provenientes de creches situadas em área

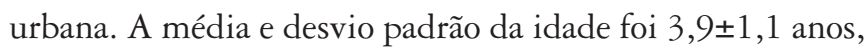
com $80,8 \%$ dos avaliados entre três e cinco anos.

O perfil antropométrico das crianças é apresentado na Tabela 1 , destacando-se a ocorrência de $6,1 \%$ de obesidade e 6,8\% de baixa estatura, de acordo com os indicadores P/E e E/I, respectivamente.

As médias e desvios padrão de peso e estatura das crianças provenientes de creches urbana e rural foram de 16,9 $93,2 \mathrm{e}$ $16,8 \pm 3,4 \mathrm{~kg}$, e $1,03 \pm 0,08$ e $1,02 \pm 0,08 \mathrm{~m}$, respectivamente, não havendo diferença estatística. Entre os sexos também não foram observadas diferenças, sendo as médias e desvios padrão

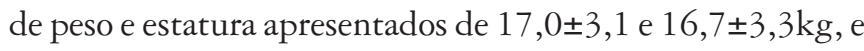
$1,03 \pm 0,08$ e $1,02 \pm 0,08 \mathrm{~m}$ para o sexo masculino e feminino, respectivamente.

A avaliação antropométrica, segundo o sexo e a área de localização das creches, encontra-se descrita nas Tabelas 2 e 3 , respectivamente. Quando comparados os dados obtidos entre os sexos, observou-se maior prevalência de estatura adequada, segundo o índice E/I, no sexo feminino ( $p=0,041$, não havendo diferença estatística entre os gêneros para a prevalência de desnutrição e obesidade, segundo o índice P/E.

A Tabela 4 apresenta os dados antropométricos segundo as faixas etárias, observando-se maior prevalência de baixa

Tabela 1 - Perfil antropométrico de crianças em creches públicas do município de Bezerros (PE) segundo os indicadores estatura/idade e peso/estatura

\begin{tabular}{lcrrr}
\hline \multirow{2}{*}{ Índice antropométrico } & \multicolumn{2}{c}{ Estatura/idade } & \multicolumn{2}{c}{ Peso/estatura } \\
\cline { 2 - 5 } & $\mathbf{n}$ & \multicolumn{1}{c}{$\%$} & $\mathbf{n}$ & $\%$ \\
\hline Baixa estatura/desnutrição & 50 & 6,8 & 5 & 0,7 \\
Risco de comprometimento estatural/nutricional & 155 & 21,1 & 60 & 8,2 \\
Adequada estatura/eutrofia & 530 & 72,1 & 625 & 85,0 \\
Obesidade & - & - & 45 & 6,1 \\
\hline
\end{tabular}


Tabela 2 - Perfil antropométrico de crianças em creches públicas do município de Bezerros (PE), segundo o sexo

\begin{tabular}{lccc}
\hline & \multicolumn{2}{c}{ Prevalência (\%) } & \multirow{2}{*}{ Valor de $\boldsymbol{p}$} \\
\cline { 2 - 3 } Índice antropométrico & Masculino & Feminino & \\
\cline { 2 - 3 } & $\mathbf{n = 3 9 5}$ & $\mathbf{n}=\mathbf{3 4 0}$ & \\
\hline Estatura/ldade & 6,3 & 7,4 & \\
Baixa estatura & 25,1 & 16,5 & \\
Risco comprometimento estatural & 68,6 & 76,2 & 0,324 \\
Adequada estatura & & & \\
Peso/Estatura & 1,0 & 0,3 & \\
Desnutrição & 7,6 & 8,8 & \\
Risco nutricional & 86,3 & 83,5 & \\
Eutrofia & 5,1 & 7,4 & \\
Obesidade & & \\
\hline
\end{tabular}

Tabela 3 - Perfil antropométrico de crianças em creches públicas do município de Bezerros (PE), segundo a localização da creche

\begin{tabular}{lccc}
\hline & \multicolumn{2}{c}{ Prevalência (\%) } \\
\cline { 2 - 3 } Índice antropométrico & Urbana & Rural & \multirow{2}{*}{$\mathbf{n}=\mathbf{2 0 1}$} \\
\cline { 2 - 3 } & $\mathbf{n = 5 3 4}$ & & \\
\hline Estatura/idade & & & \\
Baixa estatura & 7,7 & 4,5 & \\
Risco comprometimento estatural & 21,3 & 20,4 & \multirow{2}{*}{0,438} \\
Adequada estatura & 70,9 & 75,1 & \\
Peso/estatura & & & \\
Desnutrição & 0,9 & 0 & \\
Risco nutricional & 8,2 & 8,0 & \\
Eutrofia & 85,2 & 84,6 & \\
Obesidade & 5,6 & 7,5 & \\
\hline
\end{tabular}

Tabela 4 - Perfil antropométrico de crianças em creches públicas do município de Bezerros (PE), segundo a faixa etária

\begin{tabular}{|c|c|c|c|c|}
\hline \multirow{3}{*}{ Índice antropométrico } & \multicolumn{3}{|c|}{ Prevalência (\%) } & \multirow{3}{*}{ Valor de $p$} \\
\hline & $0-2$ anos & 3-5 anos & $>5$ anos & \\
\hline & $n=104$ & $\mathrm{n}=594$ & $\mathrm{n}=37$ & \\
\hline Estatura/idade & & & & $<0,001$ \\
\hline Baixa estatura & 4,8 & 6,4 & 18,9 & \\
\hline Risco comprometimento estatural & 14,4 & 21,0 & 40,5 & \\
\hline Adequada estatura & 80,8 & 72,5 & 42,5 & \\
\hline Peso/estatura & & & & 0,248 \\
\hline Desnutrição & 1,0 & 0,7 & 0 & \\
\hline Risco nutricional & 8,7 & 8,2 & 5,4 & \\
\hline Eutrofia & 88,5 & 83,8 & 94,6 & \\
\hline Obesidade & 1,9 & 7,2 & 0 & \\
\hline
\end{tabular}


estatura, segundo o índice E/I, nas crianças maiores de cinco anos.

Quanto ao déficit de peso para idade (P/I), foi encontrada uma prevalência de 3,5\% na população de estudo: 4,3\% nas crianças de creches de área urbana e $1,5 \%$ nas crianças de área rural. Calculando-se a razão entre a prevalência de obesidade (escore $Z$ de $P / E \geq+2$ ) e de déficit de peso (escore $Z$ $\mathrm{P} / \mathrm{I}<-2$ ), verificou-se que esta razão foi de 1,7:1 na população estudada, sendo 1,3:1 e 5:1 para as crianças do meio urbano e rural, respectivamente. Das crianças que apresentaram baixa estatura (E/I), $10 \%$ apresentaram obesidade.

\section{Discussão}

A obesidade e a baixa estatura aparecem como os principais problemas nutricionais relativos aos dados antropométricos avaliados, corroborando as últimas pesquisas nacionais ${ }^{(15-17)}$. A prevalência de baixa estatura $(6,8 \%)$ encontra-se acima do esperado para uma população com boas condições de saúde e nutrição $(2,3 \%)^{(6,7)}$. Os valores encontrados são similares aos resultados de outros estudos realizados no Brasil, nos quais a baixa estatura é o problema nutricional de maior relevância $^{(3,6,7,18)}$.

A prevalência de desnutrição $(0,7 \%)$ foi inferior à esperada, com resultados similares aos de estudos com crianças da mesma faixa etária em locais com melhor IDH do que o município estudado ${ }^{(1,18,19)}$. O indicador $\mathrm{P} / \mathrm{E}$ está relacionado à hipotrofia ou magreza e expressa uma desarmonia no processo de crescimento, representando um processo grave e atual de fome ${ }^{(14)}$ na ausência de comprometimento estatural associado, observando-se nos resultados desse estudo que tal forma de desnutrição é rara nesse grupo.

Em relação ao comprometimento estatural, a prevalência de baixa estatura $(21,1 \%)$ foi superior à esperada $(13,6 \%)^{(13)}$, enquanto o escore $Z$ de peso para estatura entre -2 e - $1(8,2 \%)$ encontra-se dentro do esperado para a população de referência. Esses dados estão de acordo com os encontrados por Silva $e t$ $a^{(13)}$ que, ao analisarem dados da Pesquisa Nacional de Saúde e Nutrição (PNSN, 1989) constituída por 10.667 crianças menores de seis anos, encontraram prevalência de risco de comprometimento estatural e nutricional de 24,6 e 9,7\%, respectivamente.

Assim, os resultados referentes à baixa estatura e à desnutrição são compatíveis com outros estudos brasileiros que demonstram predomínio da baixa estatura concomitante à baixa prevalência de crianças com emagrecimento extremo $^{(6,7,19,20)}$.
Oliveira $e t a^{(21)}$, em 2008, ao avaliarem o estado nutricional de 34 crianças de um a sete anos em Laranjal (PR), município com IDH de 0,651, similar ao de Bezerros (PE), encontraram prevalências de baixo peso e baixa estatura superiores às deste estudo: 20,6 e $11,8 \%$, respectivamente. Comportamento diferente foi observado quando comparadas as prevalências de obesidade, sendo verificado 2,9\% no município de Laranjal.

A prevalência de obesidade aqui observada ultrapassou a média de outros estudos: Monteiro e Conde ${ }^{(7)}$ encontraram $4 \%$ na cidade de São Paulo e Taddei ${ }^{(22)}$ notou prevalências nacionais de 5,5 e 4,1\% em menores de cinco anos avaliados nos inquéritos de 1989 e 1996, respectivamente. O aumento de peso de crianças nos últimos anos é enfatizado por vários autores $^{(1,19,20)}$. No Brasil, a rápida diminuição das taxas de desnutrição associada ao aumento das taxas de obesidade tem ocorrido em curto intervalo de tempo, agregando novas preocupações no âmbito das políticas públicas que envolvem os cuidados alimentares e nutricionais das crianças. É preciso destacar que o maior risco, em longo prazo, da obesidade infantil é sua persistência no adulto, com todas as consequências associadas para a saúde ${ }^{(23)}$. O crescente aumento do número de indivíduos obesos parece estar mais relacionado às mudanças no estilo de vida e aos hábitos alimentares, associando-se a uma epidemia de inatividade ${ }^{(4,24)}$.

A análise de estudos mais recentes permite vislumbrar a tendência ao aumento das prevalências de sobrepeso e obesidade nas crianças brasileiras, dados concordantes com os achados nesta pesquisa. Destaca-se, entre tais pesquisas, o trabalho de Corso et a $l^{(25)}$, realizado em Florianópolis (SC), que demonstrou $6,8 \%$ de obesidade em menores de seis anos; a pesquisa realizada por Gigante $e t a^{(20)} \mathrm{em}$ Pelotas, com cerca de $10 \%$ de sobrepeso no acompanhamento de 1.273 crianças; o perfil nutricional de crianças de creches de Brasília (DF) traçado por Tuma et al ${ }^{(1)}$, que mostrou 6,1\% de obesidade, e a Pesquisa Nacional de Demografia e Saúde, que demonstrou $6,6 \%$ de obesidade em crianças menores de cinco anos ${ }^{(15)}$.

As crianças do setor rural são frequentemente menores e apresentam ritmo de crescimento mais lento do que aquelas que vivem em áreas urbanas em decorrência de alguns fatores, como a maior precariedade dos serviços de saúde e educação e a irregularidade do consumo de alimentos ${ }^{(13)}$; entretanto, os resultados deste estudo não apontaram diferenças no perfil nutricional das crianças do meio urbano e rural possivelmente devido ao efeito protetor que as creches exercem sobre o estado nutricional das crianças. 
Crianças do sexo feminino apresentaram uma prevalência de baixa estatura discretamente superior. Entretanto, ao analisar o risco nutricional para esse indicador (risco de comprometimento estatural), observou-se que as crianças do sexo masculino foram mais acometidas, sendo mais elevado o percentual de adequação estatural para o sexo feminino. Esse achado corrobora os de Guimarães e Barros, em 2001, que encontraram médias de escores $Z$ mais baixas entre os meninos nos três índices (peso/idade, estatura/idade e peso/estatura $)^{(8)}$. Sabe-se que o gênero é um fator biológico que interfere no estado nutricional, determinando o crescimento e o estoque energético diferenciado entre o sexo feminino e o masculino, ou seja, menor crescimento estatural e acúmulo energético na forma de gordura para as meninas e maior crescimento estatural e maior oferta proteica para os meninos ${ }^{(26)}$. A relevância mais evidente de déficits antropométricos no sexo masculino pode estar associada à sua maior suscetibilidade às condições inadequadas de vida ${ }^{(8)}$.

Foram observadas menores prevalências de obesidade e baixa estatura nas crianças menores de dois anos, quando comparadas com as crianças na faixa etária dos três aos cinco anos e com a análise de todas as crianças deste estudo, sem agrupar em faixa etária. A baixa prevalência de obesidade encontrada entre os lactentes do município de Bezerros (PE) contrasta com inquéritos antropométricos brasileiros de 1989 e 1996, nos quais se verificou o aumento da prevalência de obesidade em crianças menores de dois anos nas regiões Norte e Nordeste (de 2,5 para 4,5\%) $)^{(22)}$, e com as estimativas da PNDS (2006), que atesta prevalência de obesidade em $6,7 \%$ das crianças menores de um ano e $6,0 \%$ nas crianças entre um e dois anos ${ }^{(15)}$. Porém, devido ao caráter transversal deste estudo e à ausência de análise de fatores possivelmente determinantes do estado nutricional infantil, como aleitamento materno, peso ao nascer, escolaridade materna, condições de moradia e saneamento, renda familiar etc., não foi possível traçar uma relação de causa e efeito para esses resultados. No entanto, sugere-se que as políticas sociais da última década, com avanço na Estratégia Saúde da Família, aumento da cobertura de programas de transferência de renda (Bolsa Família) e acesso à creche ${ }^{(15)}$, tenham atuado como fator protetor do estado nutricional das crianças menores de dois anos do município de Bezerros (PE).

Crianças que frequentam creches obtêm praticamente $70 \%$ de suas necessidades nutricionais durante o período de permanência nessas instituições e recebem alimentação durante aproximadamente dez horas do dia, o que sugere que deveriam estar mais protegidas dos distúrbios nutricionais ${ }^{(27)}$. As prevalências de obesidade e baixa estatura neste estudo, acima das esperadas, podem ser indicativas de que fatores socioeconômicos influenciaram o estado nutricional dos pré-escolares estudados, apontando a importância do acompanhamento dos desvios e riscos nutricionais, uma vez que o estado nutricional nessa faixa etária pode ser determinante para o estado nutricional na vida adulta ${ }^{(28)}$.

A razão obesidade/déficit de peso, que permite inferir sobre o avanço do predomínio da obesidade sobre a prevalência de baixo peso para idade, foi inferior à encontrada por Monteiro et $a^{(2)}$ em crianças brasileiras de maior renda $(7,6: 1)$ e por Guimarães e Barros ${ }^{(8)}$ em pré-escolares da área do centro da rede pública de Cosmópolis (SP) (8,3:1). Entretanto, os resultados deste estudo são similares aos apresentados por Guimarães e Barros ${ }^{(8)}$ quando se referem às crianças das escolas fora do centro $(2,3: 1)$. Observa-se a mesma tendência na população do estudo, com maior expressividade do processo de transição alimentar entre as crianças do meio rural $(5: 1)$.

Popkins et a ${ }^{(29)}$ analisaram inquéritos nacionais de quatro países, incluindo o Brasil, e verificaram associação positiva entre baixa estatura e obesidade. No presente estudo, das 735 crianças avaliadas, $50(6,8 \%)$ apresentaram déficit de estatura e, destas, cinco (10\%) mostraram obesidade associada, contrariando os resultados de Corso et al ${ }^{(25)}$, que identificaram associação entre estas duas variáveis em apenas $0,3 \%$ das crianças avaliadas.

A perda parcial dos dados de cerca de $30 \%$ da população de crianças matriculadas nas creches no ano de 2008 pode ter introduzido um viés de seleção. As perdas têm sido amplamente observadas na literatura, representando um dos obstáculos para a pesquisa epidemiológica. Apesar dessa limitação, os resultados apresentados são relevantes pelo grande número de crianças avaliadas e pela ausência de registros anteriores do perfil antropométrico das crianças nessa faixa etária do município de Bezerros.

Os resultados desta pesquisa apontam a necessidade de monitoramento do estado nutricional e de atuação articulada entre escolas e serviços de saúde para a prevenção e controle dos desvios nutricionais identificados. As creches escolares devem ser utilizadas como espaço estratégico para intervenção nutricional e ampliação das experiências infantis na formação de hábitos alimentares saudáveis. 


\section{Referências bibliográficas}

1. Tuma RC, Costa TH, Schmitz BA. Dietary and anthropometric assessment of three pre-schools from Brasilia, Federal District, Brazil. Rev Bras Saude Matern Infant 2005;5:419-28.

2. Monteiro CA, Mondini L, de Souza AL, Popkin BM. The nutrition transition in Brazil. Eur J Clin Nutr 1995;49:105-13.

3. Fisberg RM, Marchioni DM, Cardoso MR. Nutritional status and factors associated with stunting in children attending public daycare centers in the Municipality of São Paulo, Brazil. Cad Saude Publica 2004;20:812-7.

4. Fernandes IT, Gallo PR, Advíncula AO. Anthropometric assessment in preschool children in Mogi-Guaçu, State of São Paulo: a support for public health policies. Rev Bras Saude Matern Infant 2006;6:217-22.

5. Campos LA, Leite AJ, Almeida PC. Prevalence of overweight and obesity among adolescent students in the city of Fortaleza, Brazil. Rev Bras Saude Matern Infant 2007;7:183-90.

6. Silva MV, Sturion GL. Freqüência à creche e outros condicionantes do estado nutricional infantil. Rev Nutr 1998;11:58-68.

7. Monteiro CA, Conde WL. Secular trends in malnutrition and obesity among children in S. Paulo City, Brazil (1974-1996). Rev Saude Publica 2000;34 (Supl 6):52-61.

8. Guimarães LV, Barros MB. Differences between the nutritional status of children in public preschools and nutritional transition. J Pediatr (Rio J) 2001;77:381-6.

9. Barros AA, Barros MB, Maude GH, Ross DA, Davies PS, Preece MA. Evaluation of the nutritional status of 1st-year school children in Campinas, Brazil. Ann Trop Paediatr 1990;10:75-84.

10. Sales GC, Ferreira TR, Miranda AS, Silva AK, Campos AP, Amaral JA et al. Estado nutricional de pré-escolares de uma escola de ensino particular do município de Caratinga, MG. III Encontro de Pesquisas das IES; 2006; Minas Gerais, Brasil. p.173.

11. Brasil - IBGE. Resultados da amostra do Censo Demográfico 2000 - Malha municipal digital do Brasil: situação em 2001. Rio de Janeiro: IBGE; 2004.

12. Atlas do Desenvolvimento Humano no Brasil. Programa das Nações Unidas para o Desenvolvimento - PNUD; 2000.

13. Silva MV, Ometto AM, Furtuoso MC, Pipitone MA, Sturion GL. Access to daycare centers and the nutritional status of Brazilian children: regional differences by age group and income class. Rev Nutr 2000;13:193-9.

14. WHO. Health Organization. Physical Status: the use and interpretation of anthropometry. Report of a WHO Expert Committee. Technical report series 854. Geneva: World Health Organization; 1995.

15. Brasil. Ministério da Saúde. Pesquisa nacional de demografia e saúde da criança e da mulher (PNDS), 2006. Relatório. Brasília: PNDS, 2008.

16. Instituto Brasileiro de Geografia e Estatística (IBGE). Sociedade Civil Bem-
Estar Familiar no Brasil. Pesquisa Nacional sobre Demografia e Saúde - PNDS; 1996. Rio de Janeiro: IBGE; 1996.

17. Instituto Nacional de Alimentação e Nutrição (INAN). Pesquisa Nacional sobre Saúde e Nutrição. Perfil de Crescimento da População Brasileira de 0 a 25 anos - PNSN. Brasília: INAN; 1989.

18. Victora CG, Gigante DP, Barros AJ, Monteiro CA, Onis M. Estimating the prevalence of height for age deficits based on the prevalence of low weight for age among Brazilian children. Rev Saude Publica 1998;32:321-7.

19. Zöllner CC, Fisberg RM. Nutritional status and relationship with biological, social and demographical issues of children attending daycare centers of the local government of the city of São Paulo. Rev Bras Saude Matern Infant 2006;6:319-28.

20. Gigante DP, Victora CG, Araújo CL, Barros FC. Trends in the nutritional profile of children born in 1993 in Pelotas, Rio Grande do Sul, Brazil: longitudinal analyses. Cad Saude Publica 2003;19 (Suppl 1):141-7.

21. Oliveira WL, Oliveira FL, Amancio OM. Nutritional status, hematological and serum levels of iron in preschool children from cities with different child development indexes. Rev Paul Pediatr 2008;26:225-30.

22. Taddei JA, Colugnati FA, Rodrigues EM, Sigulem DM, Ancona-Lopez F. Desvios nutricionais em menores de 5 anos. São Paulo: Unifesp; 2002.

23. Guo SS, Roche AF, Chumlea WC, Gardner JD, Siervogel RM. The predictive value of childhood body mass index values for overweight at age $35 \mathrm{y}$. Am J Clin Nutr 1994;59:810-9.

24. Torriente GM, Molina DC, Díaz Y, Fernández AT, Argüelles XH. Obesidad en la infancia: diagnóstico y tratamiento. Rev Cubana Pediatr 2002;74:233-9.

25. Corso AC, Viteritte PL, Peres MA. Prevalence of overweight and its association with the area of residence among 6-year-old children enrolled in public childcare centers in Florianópolis, Brazil. Rev Bras Epidemiol 2004;7:201-9.

26. Santos AL, Leão LS. Anthropometric profile of preschool children of a daycare center in Duque de Caxias, Rio de Janeiro, Brazil. Rev Paul Pediatr 2008;26:218-24.

27. Biscegli TS, Corrêa CE, Romera J, Candido AB. Nutritional status and iron deficiency among children enrolled in a day care center before and after 15 months of nutritional management. Rev Paul Pediatr 2008;26:124-9.

28. Damaceno RJ, Martins PA, Devincenzi MU. Nutritional status of children assisted in public health care settings of the city of Santos, São Paulo, Brazil. Rev Paul Pediatr 2009;27:139-47.

29. Popkin BM, Richards MK, Monteiro C. Stunting is associated with overweight in children of four nations that are undergoing the nutrition transition. J Nutr 1996;126:3009-16. 